\title{
Racial Disparities in 30-Day Outcomes Following Index Admission for COVID-19
}

\begin{abstract}
Vivek Nimgaonkar ${ }^{1 \dagger}$, Jeffrey C. Thompson ${ }^{2 \dagger}$, Lauren Pantalone ${ }^{3}$, Tessa Cook ${ }^{3}$, Despina Kontos ${ }^{3}$, Anne Marie McCarthy ${ }^{4 * \neq}$ and Erica L. Carpenter ${ }^{5 \neq}$

${ }^{1}$ Perelman School of Medicine, University of Pennsylvania, Philadelphia, PA, United States, ${ }^{2}$ Division of Pulmonary, Allergy, and Critical Care Medicine, Thoracic Oncology Group, Department of Medicine, University of Pennsylvania Perelman School of Medicine, Philadelphia, PA, United States, ${ }^{3}$ Department of Radiology, University of Pennsylvania Perelman School of Medicine, Philadelphia, PA, United States, ${ }^{4}$ Department of Biostatistics, Epidemiology and Informatics, University of Pennsylvania Perelman School of Medicine, Philadelphia, PA, United States, ${ }^{5}$ Division of Hematology-Oncology, Department of Medicine, University of Pennsylvania Perelman School of Medicine, Philadelphia, PA, United States
\end{abstract}

\section{OPEN ACCESS}

Edited by:

Yann Joly,

McGill University, Canada

Reviewed by:

Lorraine Dean,

Johns Hopkins University,

United States

Gratien Dalpe,

McGill University, Canada

*Correspondence:

Anne Marie McCarthy

annemcc@pennmedicine.upenn.edu

†These authors have contributed equally to this work and share first authorship

¥These authors have contributed equally to this work and share last authorship

Specialty section:

This article was submitted to Infectious Diseases - Surveillance,

Prevention and Treatment, a section of the journal Frontiers in Medicine

Received: 30 July 2021 Accepted: 07 October 2021 Published: 02 November 2021

Citation:

Nimgaonkar V, Thompson JC, Pantalone L, Cook T, Kontos D, McCarthy AM and Carpenter EL (2021) Racial Disparities in 30-Day Outcomes Following Index Admission for COVID-19. Front. Med. 8:750650.

doi: 10.3389/fmed.2021.750650
We investigated racial disparities in a 30-day composite outcome of readmission and death among patients admitted across a 5-hospital health system following an index COVID-19 admission. A dataset of 1,174 patients admitted between March 1, 2020 and August 21, 2020 for COVID-19 was retrospectively analyzed for odds of readmission among Black patients compared to all other patients, with sequential adjustment for demographics, index admission characteristics, type of post-acute care, and comorbidities. Tabulated results demonstrated a significantly greater odds of 30-day readmission or death among Black patients (18.0\% of Black patients vs. $11.3 \%$ of all other patients; Univariate Odds Ratio: 1.71, $p=0.002)$. Sequential adjustment via logistic regression revealed that the odds of 30-day readmission or death were significantly greater among Black patients after adjustment for demographics, index admission characteristics, and type of post-acute care, but not comorbidities. Stratification by type of post-acute care received on discharge revealed that the same disparity in odds of 30-day readmission or death existed among patients discharged home without home services, but not those discharged to home with home services or to a skilled nursing facility or acute rehab facility. Collectively, the findings suggest that weighing comorbidity burdens in post-acute care decisions may be relevant in addressing racial disparities in 30-day outcomes following discharge from an index COVID-19 admission.

\section{Keywords: readmission, COVID-19, racial disparity, comorbidity, post-acute care}

\section{INTRODUCTION}

Racial disparities in infection, hospitalization, and mortality from coronavirus disease 2019 (COVID-19) have been documented, particularly for Black Americans (1). Recent studies have investigated readmission rates following hospitalization for $\operatorname{COVID}-19(2,3)$, showing that most readmissions occur within 10 days of discharge (4). There is limited data on the effect of postacute care on racial disparities in 30-day readmissions. Evaluating determinants of racial disparities in COVID-19 readmissions could enable targeted interventions to address inequities. Thus, we analyzed 30-day outcomes of COVID-19 patients surviving to discharge across a 5-hospital health system. 


\section{MATERIALS AND METHODS}

This retrospective study of 30-day readmission or death among patients hospitalized between $3 / 1 / 2020$ and $8 / 21 / 2020$ for COVID-19 was conducted within the University of Pennsylvania Health System and approved by the University's Institutional Review Board. Patients were identified using a dataset of all patients with an order for any COVID-19 test and chest imaging completed within the health system. Only patients admitted within 14 days of placement on the health system's COVID19 positive registry and with primary diagnosis ICD-10 codes consistent with COVID-19 were included. Patient characteristics from the medical record were compared by race using the chi-squared and Mann-Whitney tests. Logistic regression of 30-day outcome was sequentially adjusted for demographics, index admission characteristics, type of post-acute care, and comorbidities (5). We tested the interaction of race with postacute care using the likelihood-ratio test and performed stratified regression analyses. All analyses were completed on Stata MP Version 15.1 (StataCorp).

\section{RESULTS}

Of 1,461 admitted COVID-19 patients, 1,174 survived to discharge. 625 patients were Black (53.0\%); a majority of the remaining patients were White $(n=427,37.1 \%)$, and $10 \%$ reported Hispanic ethnicity. Black patients were younger, more likely female, had different forms of insurance, and a greater burden of comorbidities. Index admission length and frequency of mechanical ventilation were similar between Black patients and patients of other races, but the distribution of post-acute care on discharge differed (Table 1).

Amongst all discharged patients, 176 patients (15.0\%) were readmitted or died within 30 days: 116 Black patients (18.0\%) vs. 60 patients of other races (11.3\%) (Table 1). A univariate odds ratio of 30-day readmission or death among Black patients was 1.71 (CI: $1.23-2.40, p=0.002$ ) without meaningful change on sequential adjustment for demographics, index admission, and post-acute care (Table 2, Models 1-4). Addition of comorbidities, however, attenuated the odds ratio for race with 30-day readmission or death, and it was no longer statistically significant (Table 2, Model 5).

When stratified by post-acute care, univariate odds of readmission or death were significantly greater for Black patients discharged to home without services $(\mathrm{OR}=2.12,95 \%$ CI: 1.19 $3.78, p=0.010)$ but not to home health care ( $\mathrm{OR}=1.18$, CI: $0.64-$ $2.17, p=0.594$ ) or skilled nursing or acute rehab facilities ( $\mathrm{OR}=$ 1.70 , CI: $0.87-3.32, p=0.119)$. However, the interaction between Black race and post-acute care was not statistically significant $(p$-interaction $=0.309)$.

Multiple sensitivity analyses were performed. With 30-day readmission as the sole endpoint and patients who died in the timeframe excluded, the same trend in greater odds of readmission among Black patients was observed on serial adjustment for contributory variables, where odds of readmission were no longer significant after adjustment for comorbidities. Identical trends were observed on sensitivity analyses using eight individual comorbidities instead of the Charlson Comorbidity Index, excluding readmissions to other health systems, and including COVID-19 registry patients with any ICD-10 code.

\section{DISCUSSION}

Black patients were more likely to be readmitted or die at 30 days following admission for COVID-19 across an urban health system after adjusting for demographics, post-acute care, and index admission characteristics. To our knowledge, this is the first study to note a racial disparity in 30-day outcomes following COVID-19 hospitalization. This is consistent with disparities in readmissions seen for conditions that frequently cause hospitalizations, such as myocardial infarctions, decompensated heart failure, and pneumonia (6). For these conditions, disparities in readmissions were also related to the sites of care such that hospitals serving larger minority populations similar to the population in our study had larger disparities in readmission rates. Analysis in 2018 following the institution of Medicare's Hospital Readmission Reduction Program, which penalizes hospitals for higher than expected 30-day readmissions for acute myocardial infarction, heart failure, and pneumonia, suggest that racial disparities have persisted and may have widened for non-targeted conditions at safety net hospitals that care for larger populations of Black patients (7). While the site of care has been associated with racial disparities in readmissions, there are other structural inequities that could contribute to greater readmissions among Black patients. In a national study of readmissions in diabetic patients, other demographic factors, most notably household income, also contributed to the disparity in readmissions along with the site of care (8). We lacked data on household income or other measures of socioeconomic status beyond insurance status.

It should be noted that a greater proportion of the Black patients admitted in our study were females, though sex was not found to be statistically significant in our regressions on 30day outcomes. The larger proportion of females admitted for COVID-19 among the Black population in our study could reflect a differential impact of the pandemic on the sexes across racial groups. It has been observed that COVID-19 outcomes among males and females can differ between Black and White patients, though previously presented data have described worse outcomes among Black males rather than females (9). Alternatively, the higher proportion of Black females admitted for COVID-19 in our study may be attributable in part to regional population level differences in the ratio of females to males by race above the age of 50 (10), which are due to shorter life expectancy among Black men (11).

Differences in the burden of comorbidities largely explained the racial difference in 30-day outcomes. Greater comorbidities among Black patients have previously been attributed to the effects of structural racism on health and limited access to care (12). Though analyses of racial disparities in readmissions during epidemics and pandemics are limited, there is a robust literature documenting the disproportionate impacts of disease outbreaks on racial minorities, and thorough analysis of the 1918 
TABLE 1 | Characteristics of Black and Non-Black patients by site of discharge following index admission for COVID-19.

\begin{tabular}{|c|c|c|c|c|}
\hline & Total & Black patients & All other patients & $P$-value* \\
\hline$n$ & 1,174 & 645 & 529 & \\
\hline \multicolumn{5}{|l|}{ Racial sub-groups } \\
\hline Black (\%) & $645(54.9)$ & & & \\
\hline White (\%) & $427(36.4)$ & & & \\
\hline Other (\%) & $102(8.7)$ & & & \\
\hline \multicolumn{5}{|l|}{ Ethnicity } \\
\hline Hispanic & $124(10.6)$ & $22(3.4)$ & $102(19.3)$ & \\
\hline \multicolumn{5}{|l|}{ Additional demographics } \\
\hline Age, median (yrs, IQR) & $62(49,74)$ & $61(48,71)$ & $63(50,77)$ & 0.022 \\
\hline Female, $n(\%)$ & $566(48.2)$ & $340(52.7)$ & $226(42.7)$ & 0.001 \\
\hline \multicolumn{5}{|l|}{ Insurance } \\
\hline Private (\%) & $282(24.0)$ & $159(24.7)$ & 123 (23.3) & $<0.001$ \\
\hline Medicare (\%) & 555 (47.3) & $290(45.0)$ & $265(50.1)$ & \\
\hline Medicaid (\%) & $223(19.0)$ & $154(23.9)$ & $69(13.0)$ & \\
\hline Uninsured (\%) & $38(3.2)$ & $8(1.2)$ & $30(5.7)$ & \\
\hline Unknown (\%) & $76(6.5)$ & $34(5.3)$ & $42(7.9)$ & \\
\hline \multicolumn{5}{|l|}{ Comorbidities } \\
\hline Charlson comorbidity index, mean (SD) & $3.2(3.3)$ & $3.9(3.6)$ & $2.3(2.7)$ & $<0.001$ \\
\hline Type 2 diabetes mellitus (\%) & $437(37.2)$ & $289(44.8)$ & $148(28.0)$ & $<0.001$ \\
\hline Chronic obstructive pulmonary disease (\%) & $124(10.6)$ & $80(12.4)$ & $44(8.3)$ & 0.023 \\
\hline Congestive heart failure (\%) & $243(20.7)$ & $156(24.2)$ & $87(16.4)$ & 0.001 \\
\hline Chronic kidney disease (\%) & $197(16.8)$ & $147(22.8)$ & $50(9.5)$ & $<0.001$ \\
\hline Hypertension (\%) & $786(67.0)$ & $483(74.9)$ & $303(57.3)$ & $<0.001$ \\
\hline Cancer (\%) & $219(18.7)$ & $124(19.2)$ & $95(18.0)$ & 0.579 \\
\hline History of cerebrovascular infarction (\%) & $189(16.1)$ & $128(19.8)$ & $61(11.5)$ & $<0.001$ \\
\hline Sickle cell disease (\%) & $21(1.8)$ & $21(3.3)$ & $0(0.0)$ & $<0.001$ \\
\hline \multicolumn{5}{|l|}{ Index admission } \\
\hline Length of stay (days, IQR) & $6(3,12)$ & $5(3,12)$ & $6(3,11)$ & 0.164 \\
\hline Mech. ventilated (\%) & $152(12.9)$ & $87(13.5)$ & $65(12.3)$ & 0.542 \\
\hline \multicolumn{5}{|l|}{ Post-acute care on discharge } \\
\hline Home (without services) (\%) & $523(44.5)$ & $271(42.0)$ & $252(47.6)$ & $<0.001$ \\
\hline Home health care (\%) & $352(30.0)$ & $230(35.7)$ & $122(23.1)$ & \\
\hline SNF/acute rehab (\%) & $241(20.5)$ & $114(17.7)$ & $127(24.0)$ & \\
\hline Other (\%) & $58(4.9)$ & $30(4.7)$ & $28(5.3)$ & \\
\hline \multicolumn{5}{|l|}{ 30-day outcomes } \\
\hline Readmission (\%) & $161(13.7)$ & $105(16.3)$ & $56(10.6)$ & 0.005 \\
\hline Time to readmission (median days, IQR) & $7(3,14)$ & $6(3,15)$ & $7(3,13.5)$ & 0.9674 \\
\hline Death at 30 days (\%) & $20(1.7)$ & $14(2.2)$ & $6(1.1)$ & 0.175 \\
\hline Readmission or death 30 Days (\%) & $176(15.0 \%)$ & $116(18.0)$ & $60(11.3)$ & 0.002 \\
\hline
\end{tabular}

${ }^{*}$ All p-values are for chi-squared or Mann-Whitney tests; chi-squared tests were used for categorical variables and Mann-Whitney tests were used for continuous variables. Bold values denote $p$-values that were statistically significant at the $p<0.05$ level.

Influenza Pandemic suggests that structural inequities in access to care contributed to racial disparities in outcomes such as mortality (13). In fact prior to the COVID-19 pandemic, some had predicted that a future pandemic influenza was likely to result in worse outcomes in Black populations due to greater barriers to care and burdens of comorbidities among Black patients (14).

The racial disparity appeared most pronounced among patients discharged home without services. It has previously been noted that readmissions are driven not just by the inpatient care received, but also by the outpatient care and socioeconomic resources available after discharge (15). Post-acute care can range from home services, such as visiting nurses, to care in a facility. Without post-acute care, the effects of structural racism on access to care may be exacerbated. Our study suggests that racial disparities in 30-day outcomes may be most evident in the absence of post-acute care. Paired with the finding that comorbidities explained much of the disparity in 30day outcomes, these results suggest that weighing comorbidity burdens in post-acute care decisions may help address racial disparities in outcomes of discharged COVID-19 patients. 


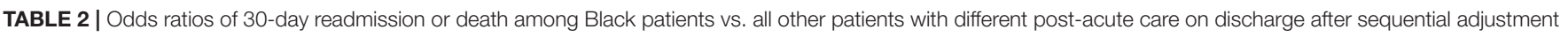
for other demographics, index admission characteristics, and comorbidities.

\begin{tabular}{|c|c|c|c|c|c|c|c|c|c|c|}
\hline & \multicolumn{2}{|l|}{$\begin{array}{l}\text { Model 1: } \\
\text { univariate* }\end{array}$} & \multicolumn{2}{|c|}{$\begin{array}{l}\text { Model 2: } \\
\text { adjusted for age, } \\
\text { sex, insurance }^{t}\end{array}$} & \multicolumn{2}{|c|}{$\begin{array}{l}\text { Model 3: additionally } \\
\text { adjusted for index length } \\
\text { of stay and ventilation }\end{array}$} & \multicolumn{2}{|c|}{$\begin{array}{l}\text { Model 4: additionally } \\
\text { adjusted for discharge } \\
\text { site }^{\S}\end{array}$} & \multicolumn{2}{|c|}{$\begin{array}{l}\text { Model 5: additionally } \\
\text { adjusted for Charlson } \\
\text { comorbidity index" }\end{array}$} \\
\hline & $\begin{array}{l}\text { Odds ratio } \\
(95 \% \mathrm{Cl})\end{array}$ & $P$-value & $\begin{array}{l}\text { Odds ratio } \\
\text { (CI) }\end{array}$ & $P$-value & $\begin{array}{l}\text { Odds ratio } \\
\text { (CI) }\end{array}$ & $P$-value & $\begin{array}{l}\text { Odds ratio } \\
\text { (CI) }\end{array}$ & $P$-value & $\begin{array}{l}\text { Odds } \\
\text { ratio }(\mathrm{Cl})\end{array}$ & $P$-value \\
\hline All patients $(n=1,174)$ & $\begin{array}{c}1.71(1.23 \\
2.40)\end{array}$ & 0.002 & $\begin{array}{c}1.81(1.28 \\
2.56)\end{array}$ & 0.001 & $\begin{array}{c}1.80(1.27 \\
2.55)\end{array}$ & 0.001 & $\begin{array}{c}1.79(1.26 \\
2.55)\end{array}$ & 0.001 & $\begin{array}{c}1.39(0.96 \\
2.01)\end{array}$ & 0.085 \\
\hline $\begin{array}{l}\text { Patients discharged } \\
\text { home without services } \\
(n=523)\end{array}$ & $\begin{array}{c}2.12(1.19 \\
3.78)\end{array}$ & 0.010 & $\begin{array}{c}2.09(1.15 \\
3.80)\end{array}$ & 0.015 & $\begin{array}{c}2.06(1.13 \\
3.76)\end{array}$ & 0.018 & & & $\begin{array}{c}1.74(0.93 \\
3.24)\end{array}$ & 0.082 \\
\hline $\begin{array}{l}\text { Patients discharged to } \\
\text { home health care }(n= \\
\text { 352) }\end{array}$ & $\begin{array}{c}1.18(0.64 \\
2.17)\end{array}$ & 0.594 & $\begin{array}{c}1.31(0.69 \\
2.49)\end{array}$ & 0.417 & $\begin{array}{c}1.28(0.67 \\
2.45)\end{array}$ & 0.457 & & & $\begin{array}{c}0.93(0.47 \\
1.86)\end{array}$ & 0.844 \\
\hline $\begin{array}{l}\text { Patients discharged to } \\
\text { SNF/acute rehab }(n= \\
\text { 241) }\end{array}$ & $\begin{array}{c}1.70(0.87 \\
3.32)\end{array}$ & 0.119 & $\begin{array}{c}2.00(0.99 \\
4.06)\end{array}$ & 0.054 & $\begin{array}{c}1.91(0.93 \\
3.90)\end{array}$ & 0.076 & & & $\begin{array}{c}1.39(0.64 \\
3.00)\end{array}$ & 0.405 \\
\hline
\end{tabular}

Model Definitions (all regressed on 30 readmission or death, bolded independent variables were significant at the $p<0.05$ level in the all patient regression).

*Model 1: Black race alone (univariate).

${ }^{\dagger}$ Model 2: Black race, sex, patient age, and insurance.

${ }^{\ddagger}$ Model 3: Black race, sex, patient age, insurance, index admission length of stay (days), index admission mechanical ventilation.

$\S$ Model 4: Black race, sex, patient age, insurance, index admission length of stay (days), index admission mechanical ventilation, post-acute care on discharge.

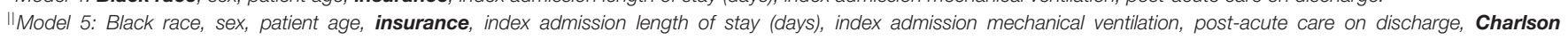
Comorbidity Index.

There are several limitations of this study. First, without information on the reasons for 30-day readmission or death, it is difficult to understand the degree to which 30-day outcomes were driven primarily by COVID-19 versus other conditions. Additionally, this study captured only deaths occurring in the 30-day period after discharge that were recorded in the electronic medical record. Consequently, it is possible that some deaths may have been missed with unclear impacts on the composite 30-day outcome of readmission and death.

Nevertheless, this study has illustrated that Black patients across an urban health system were more likely to be readmitted or die within 30 days after an index admission for COVID-19 in the initial surge of the pandemic. Existing comorbidities appeared to play an important role in explaining racial disparities, particularly among patients without post-acute care. Future studies should explore whether these findings are present in other cohorts, including at different time points in the pandemic. Validation across other cohorts would motivate interventional studies that interrogate the formal incorporation of burdens of comorbidities into the assignment of post-acute care at discharge following hospitalization for COVID-19 as a means of reducing racial disparities in readmissions.

\section{REFERENCES}

1. Mackey K, Ayers CK, Kondo KK, Saha S, Advani S, Young S, et al. Racial and ethnic disparities in COVID-19-related infections, hospitalizations, and deaths: a systematic review. Ann Intern Med. (2021) 174:36273. doi: 10.7326/M20-6306

\section{DATA AVAILABILITY STATEMENT}

The datasets presented in this article are not readily available due to the need to maintain patient confidentiality. Requests to access the de-identified datasets should be directed to annemcc@pennmedicine.upenn.edu.

\section{AUTHOR CONTRIBUTIONS}

All authors listed have made a substantial, direct and intellectual contribution to the work, and approved it for publication.

\section{FUNDING}

Work on this project was supported with funding from the Penn Center for Precision Medicine.

\section{ACKNOWLEDGMENTS}

The authors would like to thank Abigail Doucette for providing assistance with retrieval of electronic medical record data and also David Roth and Carmen Guerra for providing critical feedback.

2. Donnelly JP, Wang XQ, Iwashyna TJ, Prescott HC. Readmission and death after initial hospital discharge among patients with COVID-19 in a large multihospital system. JAMA. (2021) 325:304-6. doi: 10.1001/jama.2020.21465

3. Lavery AM, Preston LE, Ko JY, Chevinsky JR, DeSisto CL, Pennington AF, et al. Characteristics of hospitalized COVID-19 patients discharged and experiencing same-hospital readmission - 
United States, March-August 2020. MMWR Morb Mortal Wkly Rep. (2020) 69:1695-9. doi: 10.15585/mmwr.mm6945e2

4. Yeo I, Baek S, Kim J, Elshakh H, Voronina A, Lou MS, et al. Assessment of thirty-day readmission rate, timing, causes and predictors after hospitalization with COVID-19. J Intern Med. (2021) 290:157-65. doi: 10.1111/joim.13241

5. Hosmer DW, Lemeshow S. Applied Logistic Regression. New York, NY: Wiley (2000). doi: 10.1002/0471722146

6. Joynt KE, Orav EJ, Jha AK. Thirty-day readmission rates for Medicare beneficiaries by race and site of care. JAMA. (2011) 305:675-81. doi: 10.1001/jama.2011.123

7. Chaiyachati KH, Qi M, Werner RM. Changes to racial disparities in readmission rates after Medicare's Hospital readmissions reduction program within safety-net and non-safety-net hospitals. JAMA Netw Open. (2018) 1:e184154. doi: 10.1001/jamanetworkopen.2018.4154

8. Rodriguez-Gutierrez R, Herrin J, Lipska KJ, Montori VM, Shah ND, McCoy RG. Racial and ethnic differences in 30-day hospital readmissions among US adults with diabetes. JAMA Netw Open. (2019) 2:e1913249. doi: 10.1001/jamanetworkopen.2019.13249

9. Rushovich T, Boulicault M, Chen JT, Danielsen AC, Tarrant A, Richardson SS, et al. Sex disparities in COVID-19 mortality vary across US racial groups. $J$ Gen Intern Med. (2021) 36:1696-701. doi: 10.1007/s11606-021-06699-4

10. United States Census Bureau. American Community Survey. (2021). Available online at: https://www.census.gov/programs-surveys/acs/microdata.html (accessed October 4, 2021).

11. Bond MJ, Herman AA. Lagging life expectancy for black men: a public health imperative. Am J Public Health. (2016) 106:1167-9. doi: 10.2105/AJPH.2016.303251

12. Khazanchi R, Evans CT, Marcelin JR. Racism, not race, drives inequity across the COVID-19 continuum. JAMA Netw Open. (2020) 3:e2019933. doi: 10.1001/jamanetworkopen.2020.19933
13. Krishnan L, Ogunwole SM, Cooper LA. Historical insights on coronavirus disease 2019 (COVID-19), the 1918 Influenza pandemic, and racial disparities: illuminating a path forward. Ann Intern Med. (2020) 173:47481. doi: $10.7326 / \mathrm{M} 20-2223$

14. Hutchins SS, Fiscella K, Levine RS, Ompad DC, McDonald M. Protection of racial/ethnic minority populations during an influenza pandemic. Am J Public Health. (2009) 99(Suppl. 2):S261-70. doi: 10.2105/AJPH.2009.1 61505

15. Kangovi S, Grande D. Hospital readmissions-not just a measure of quality. JAMA. (2011) 306:1796-7. doi: 10.1001/jama.201 1.1562

Conflict of Interest: The authors declare that the research was conducted in the absence of any commercial or financial relationships that could be construed as a potential conflict of interest.

Publisher's Note: All claims expressed in this article are solely those of the authors and do not necessarily represent those of their affiliated organizations, or those of the publisher, the editors and the reviewers. Any product that may be evaluated in this article, or claim that may be made by its manufacturer, is not guaranteed or endorsed by the publisher.

Copyright () 2021 Nimgaonkar, Thompson, Pantalone, Cook, Kontos, McCarthy and Carpenter. This is an open-access article distributed under the terms of the Creative Commons Attribution License (CC BY). The use, distribution or reproduction in other forums is permitted, provided the original author(s) and the copyright owner(s) are credited and that the original publication in this journal is cited, in accordance with accepted academic practice. No use, distribution or reproduction is permitted which does not comply with these terms. 